\title{
Eisensubstitution bei Crohn und Colitis
}

Ein häufiges Begleitsymptom bei Patienten mit chronisch entzündlicher Darmerkrankung ist die Eisenmangelanämie. Nach den Ergebnissen einer neueren Studie genügt in der Regel eine orale Eisensubstitution, die auch meist gut vertragen wird.

- Im Rahmen dieser Studie wurde bei Patienten mit einer chronisch entzündlichen Darmerkrankung und begleitender Eisenmangelanämie eine Eisensubstitution eingeleitet. Bei einem Hb-Wert $>10 \mathrm{~g} / \mathrm{dl}$ erhielten die betroffenen Patienten orales Eisen, bei einem Hb-Wert $<10 \mathrm{~g} / \mathrm{dl}$ wurde das Eisen intravenös gegeben. Patienten, die die orale Eisentherapie nicht vertrugen, erhielten ebenfalls eine intravenöse Eisensubstitution.

Insgesamt wurden von den 100 Patienten mit einer chronisch entzündlichen Darmerkrankung (59 mit Morbus Crohn, 41 mit Colitis ulcerosa) 78 Patienten oral und 22 Patienten intravenös therapiert. Mittels der oralen Eisensubstitution konnte bei 89\% der Patienten eine Normalisierung des $\mathrm{Hb}$-Wertes erreicht werden, mittels intravenöser Eisenbehandlung bei $77 \%$.

Bei keinem Patienten wurde eine Verschlechterung der Erkrankungsaktivität dokumentiert. Die orale Eisenbehandlung wurde nur von 5,1\% der Patienten nicht vertragen. Unerwünschte Ereignisse wurden unter der intravenösen Eisentherapie nicht beobachtet.

\section{Kommentar}

Das Ergebnis dieser Studie ist eindeutig: Besteht bei einem Patienten mit einer chronisch entzündlichen Darmerkrankung eine Eisenmangelanämie, empfiehlt sich zunächst immer die orale Substitution. Nur wenn diese nicht vertragen wird oder bei sehr ausgeprägter Anämie, sollte die intravenöse Eisengabe bevorzugt werden. Beide Applikationswege sind sicher und haben keinen negativen Einfluss auf den Krankheitsverlauf. Insgesamt dürften betroffene Patienten durch die Eisensubstitution im Hinblick auf ihre Lebensqualität und Leistungsfähigkeit profitieren.

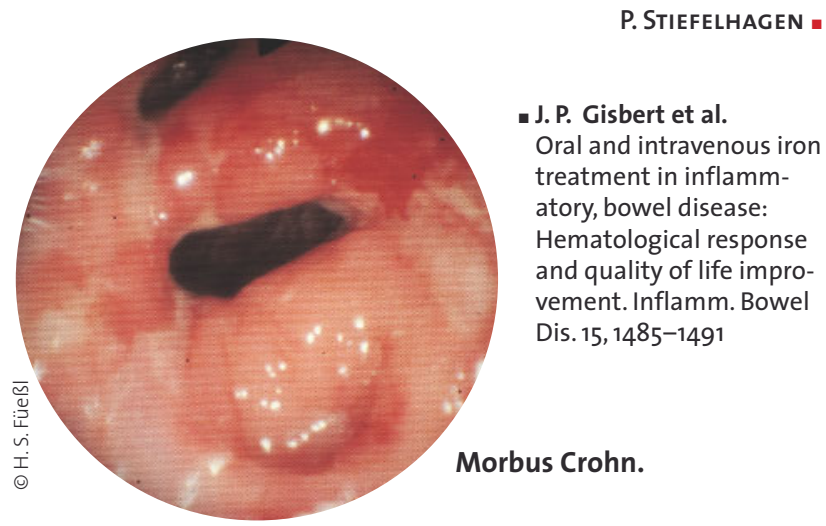

